Instituto Internacional de Investigación y Desarrollo Tecnológico Educativo INDTEC, C.A.

DOI: $h$ ttps://doi.org/10.29394/Scientific.issn.2542-2987.2020.5.15.20.384-404

OAI-PMH: http://www.indteca.com/ojs/index.php/Revista Scientific/oai

Ensayo Original / Original Essay

\title{
Pentagrama Didáctico: Una Aproximación Praxiológica de la Gestión del Conocimiento
}

Autor: Pedro Jacobo Caldera Tovar Universidad Nacional Experimental de Yaracuy, UNEY drjacobocaldera@gmail.com

Yaracuy, Venezuela https://orcid.org/0000-0003-3323-3823

Resumen

La presente producción intelectual, tiene como propósito describir el pentagrama didáctico y su aproximación praxiológica de la gestión del conocimiento que facilitan el proceso de aprendizaje, individual y colectivo. Enmarcada en el podio del constructivismo y la acción comunicativa con un lenguaje artístico musical que invita al mejoramiento permanente. Como reflexión: Preparen sus mentes, piensen en grande, no limiten sus actividades intelectuales, científicas, artísticas o de cualquier oficio, porque del conocimiento teórico y práctico, su dominio, utilización y aptitud, dependerá la evaluación o calificación obtenida.

Palabras clave: gestión; conocimiento; aprendizaje.

Cómo citar este ensayo:

Caldera, P. (2020). Pentagrama Didáctico: Una Aproximación Praxiológica de la Gestión del Conocimiento. Revista Scientific, 5(15), 384-404, e-ISSN: 2542-2987. Recuperado de: https://doi.org/10.29394/Scientific.issn.2542-2987.2020.5.15.20.384-404

Fecha de Recepción:

23-08-2019
Fecha de Aceptación:

25-11-2019
Fecha de Publicación:

05-02-2020 


\title{
Teaching Pentagram: A Praxiological Approach to Knowledge Management
}

\begin{abstract}
The purpose of this intellectual production is to describe the teaching staff and its praxiological approach to knowledge management that facilitate the learning process, individual and collective. Framed on the podium of constructivism and communicative action with a musical artistic language that invites permanent improvement. As a reflection: Prepare your minds, think big, do not limit your intellectual, scientific, artistic or any other activity, because on the theoretical and practical knowledge, your mastery, use and aptitude, the evaluation or qualification obtained will depend.
\end{abstract}

Keywords: management; knowledge; learning.

How to cite this essay:

Caldera, P. (2020). Teaching Pentagram: A Praxiological Approach to Knowledge Management. Revista Scientific, 5(15), 384-404, e-ISSN: 2542-2987. Recovered from: https://doi.org/10.29394/Scientific.issn.2542-2987.2020.5.15.20.384-404

Date Received:

23-08-2019
Date Acceptance:

25-11-2019
Date Publication:

05-02-2020 


\section{Introducción}

Durante muchos años, dedicando horas a las lecturas, llegaron momentos de inspiración y reflexionas sobre los elementos presentes en la pedagogía más allá de la filosofía como amor a la sabiduría, se me ocurrió una mañana el presente título: pentagrama didáctico: una aproximación praxiológica de la gestión del conocimiento. Mientras el Ministerio del Poder Popular para la Educación de Venezuela, resuena el término cuarteta donde involucran en la corresponsabilidad de enseñar y aprender a los docentes, familiares, estudiantes y miembros de la comunidad en general, me parece que dejan por fuera un elemento de innovación al sistema educativo: el currículo que debe ser actualizado periódicamente para no perder vigencia. A continuación, describo los elementos del pentagrama didáctico, sus cinco líneas principales:

1.- Estudiante: como razón de ser de todos los procesos sociales, científicos, artísticos, deportivos, culturales, económicos y pedagógicos, cargados de talentos humanos, vocaciones e inclinaciones por áreas del conocimiento, capaces de aplicar competencias a sus actividades para demostrar dominios adquiridos durante su corta vida, incluyendo la autodidáctica positiva (autoaprendizaje, ir más allá de los contenidos de clases, guataca) y negativa (errores de posturas, vicios, copias, imitar, actividades y procesos propios cargados de infracciones).

2.- Currículo: Material de apoyo más importante del docente, el cual debe ser revisado periódicamente con la finalidad de mantener su vigencia en el momento histórico (tiempo y espacio). Ofrece una gama de propósitos u objetivos, métodos, estrategias, actividades tomando como referencia los niveles y modalidades, los aspectos científicos, artísticos, profesionales y de oficios (manualidades, mecánica, agricultura, dibujo, entre otros).

3.- Docente: como ejecutor del currículo con sus estudiantes, es quien debe diagnosticar los intereses y necesidades para planificar proyectos de 
aprendizaje (PA), planes de acción, clases, actividades especiales, reuniones, citaciones, en pro de cumplir metas a corto, mediano y largo plazo. Se debe apropiar de la didáctica, gestionar el conocimiento, aplicar la praxiología que combina los aspectos teóricos con los prácticos. Guía los procesos de forma natural, corrigiendo errores u omisiones, repitiendo el número de veces que sea necesaria la actividad, explicar, orientar, promover, motivar y evaluar durante y después de las jornadas. Cumplir los procesos administrativos (llenado correcto de planillas, formatos, boletas, informes, constancias, permisos, corrección de evaluaciones, citaciones, actas, entre otras).

4.- Escuela: como el lugar donde se desarrolla el proceso educativo formal, con espacios naturales para el desarrollo físico e intelectual, ofrece servicios tales como aulas, laboratorio, biblioteca, cancha, cantina, comedor, según sus posibilidades. Bajo la denominación de escuela, colegio, liceo, unidad educativa, pública, privada o mixta (subsidiada). Debe poseer el proyecto educativo integral comunitario (PEIC), normas de convivencia actualizadas, permiso de funcionamiento (sólo colegios privados), áreas verdes, áreas recreativas, según su infraestructura.

5. La familia: nuclear, funcional o no, donde cada uno de los miembros aporta aspectos positivos, negativos, interesantes, financieros, sentimentales, religiosos, culturales, valores, principios, entre otros, todos moldean la personalidad, individualidad, comportamientos y conductas de cada uno de sus miembros.

6.- Comunidad: representa el contexto en la cual se desenvuelve el estudiante, donde vive, el entorno social, los entes involucrados en los alrededores del plantel, aquí es válido aplicar el análisis DAFO, también conocido como análisis FODA (Debilidades, Amenazas, Fortalezas, Oportunidades), para conocer las fortalezas, oportunidades, debilidades y amenazas que rodea la escuela, los organismos que hacen vida activa en las cercanías de la institución los cuales son corresponsables de muchas 
actividades.

Englobando lo antes expuesto, el pentagrama como tapiz musical en la cual se escribe todas las notas, claves, figuras, valores, signos, títulos, autores, tiempos, entre otras, es la base como el lienzo para la pintura, en esta combinación con la didáctica que permite encaminar procesos hacia la meta cognición, consolidar el proceso de enseñanza-aprendizaje, gestionar el conocimiento desde lo empírico hasta lo más profundo del campo científico, aplicando la praxiología unificar los aspectos teóricos y prácticos en actividades significativas, constructivas, innovadoras y productivas.

Es importante destacar, este preámbulo les invita a profundizar en procesos, elementos que mezclados ofrecen mayores y mejores resultados, hoy en día se habla de la producción intelectual, pero son más celosos con los productos, libros, partituras y artículos protegidos circulan por las redes sociales, defendiendo el derecho de autor, aunque nadie es dueño de la verdad, ni del conocimiento. Permitan abrir sus sentidos, afinar sus instrumentos y degustar de esta producción intelectual.

\section{Perspectiva emergente de la didáctica como componente cognitivo}

El proceso didáctico compartido por el docente permite desarrollar en sus estudiantes conocimientos, habilidades y destrezas innatas en muchos casos y otras capacidades partiendo del aprehensión del quehacer académico, experimental y cotidiano, según el Diccionario de las Ciencias de la Educación (1983), didáctica es el proceso de: "controlar, procesar, objetivar, guiar, integrar, optimizar, aplicar, lograr objetivos, personalizar, implicar docente-discente al proceso de enseñanza" (pág. 408).

Al realizar un ejercicio de inferencia sobre la cita anterior, podemos añadir que la didáctica es el proceso mismo de enseñar, donde el docente y el discente (del griego didaktiké, significa estudiante, sujeto a quien se dirige 
la enseñanza, es el que aprende), comparten experiencias intelectuales productivas para comprender y aprehender conocimientos según su nivel, grado o modalidad. La didáctica permite fortalecer habilidades y destrezas a través del hacer, descubrir y la praxiología (combinar conocimientos teóricos con prácticas dirigidas), minimizando las debilidades, limitaciones, errores y omisiones en el proceso pedagógico.

Desde la perspectiva epistemológica, la palabra didáctica fue utilizada por primera vez por Wolfgang (1929): su concepto giraba en función del "sentido y necesidad de enseñar" (pág. 65). Todos los que enseñan tienen dos factores en común un sentido lógico de querer aumentar el conocimiento y la necesidad de que sus discípulos aprendan, para ello utilizan todo un andamiaje de métodos, técnicas y recursos científicos, artísticos y de oficios.

Asimismo, Díaz (2001), emplea el término: "didáctica como una ciencia social, en constante desarrollo, puntualiza su accionar en la "epistemología, conceptualizaciones y metodología" (pág. 46). Como enfoque epistémico busca gestionar el conocimiento, develar nuevos conceptos partiendo de experiencias preliminares, guiados por una metodología innovadora, consciente y práctica.

Es importante destacar, la didáctica está en constante desarrollo, según los estudios de Madrid y Mayorga (2010): la "didáctica es el campo de acción de numerosas investigaciones de proyecciones teóricas y prácticas cuyos aportes han enriquecido el sistema de conocimiento y han determinado su carácter de ciencia en dos dimensiones la teoría y la práctica" (pág. 86). Como campo de acción permite el intercambio de conocimientos es escenarios naturales formales e informales, proyectar datos e informaciones más allá de la teoría y la práctica donde la utilidad de lo estudiado se aplica en la cotidianidad del estudiante.

De igual forma, Moreno (2004), manifiesta que:

Desde la consideración de los medios como materiales 
curriculares y didácticos la cuestión clave estará en su utilización y su selección con la intención de aplicarlos convenientemente a las distintas situaciones educativas $\mathrm{y}$, también, de aprovechar al máximo todas sus características técnicas y sus posibilidades didácticas (pág. 6).

En la actualidad aparecen datos, informaciones, programas y equipos capaces de desarrollar gran cantidad de tareas que antes eran manuales, la tecnología de información y comunicación, con sus redes sociales han roto para siempre la brecha de distancia y acceso del conocimiento entre los pueblos.

Es imperativo darle un valor agregado al pentagrama didáctico, ya mencionamos las líneas, es momento de ofrecer el resto de sus componentes; las claves para que se desarrollen los programas didácticos están presentes en: a). El Estado, cada país ofrece a sus habitantes una serie de programas sociales, respaldo presupuestario y la inversión a nivel nacional, incentivos, becas, infraestructuras, servicios educativos, niveles y modalidades según su misión y visión de futuro; b). La Religión, ofrece a los creyentes un modelo de vida, una guía espiritual, reflexiones sobre el accionar ante la sociedad, dando lo mejor de cada ser humano, en unión, paz, armonía, todo lo positivo, apoyados en un solo Dios; y c). Los Valores Humanos, presentes en cada momento de la vida.

Al inferir sobre valores humanos, como axiología pura, que permite a los individuos la sana convivencia, intercambios de saberes, cultura, gastronomía, turismo, ciencias, artes y diferentes oficios. Sus principios y normas han regido las sociedades desde la antigüedad, hombre, mujeres y niños prefieren cumplir las tradiciones para mantener viva la identidad, costumbres ancestrales, promover sus avances y compartir la sabiduría más allá de las fronteras de idioma, territorio y fe. Corresponde ahora el turno a los espacios del pentagrama didáctico, son cuatro grandes, cada uno de ellos 
involucra procesos educativos para irradiar a las sociedades en forma permanente:

El primero espacio es la educación formal, con su andamiaje de leyes, reglamentos, ordenanzas, que permiten ordenar jurídicamente el accionar de los docentes, directivos, estudiantes, representantes, el estado y la comunidad en general. A través de artículos norma la manera correcta de ejercer la docencia, incluye los errores, fallas y sanciones en las cuales podrían incurrir los involucrados, con figuras preestablecidas para el control y seguimiento de dichos procesos administrativos. Cada país estructura su sistema educativo en forma autónoma, en Venezuela la figura jurídica la conforma el Ministerio del Poder Popular para la Educación, con zonas educativas en cada estado, distritos escolares en cada municipio y supervisores en cada circuito escolar.

El segundo espacio es el aprendizaje informal, cotidiano, autodidáctico, por ensayo y error, por prácticas repetitivas, a través de la lectura, adquirido por observación directa de procesos laborales, al instruir verbalmente en una tarea a un compañero de trabajo o estudio, por consejos familiares, experiencias de vida, gran número de personas repiten la frase "la universidad de la vida". Con un programa personalizado que ofrece a cada individuo elementos para su crecimiento, desarrollo, positivo y negativo.

El tercer espacio es la producción, como resultado de múltiples procesos de pensamiento, palabras y obras, podemos citar la producción musical de los artistas, compositores, cantantes, arreglistas, ejecutantes. Producción intelectual de trabajos científicos, artículos, libros, tesis, proyectos, programas, ideas innovadoras, ponencias, conferencias, discursos, incluyendo las mismas clases. Producción cultural la responsable de dejar huellas en las sociedades a través del arte, música, teatro, cine, fotografía, poesía, literatura, arquitectura, con técnicas, estrategias y recursos distintos combinados por la mano del autor.

El cuarto espacio, no menos importante, la promoción del trabajo, 
conocimientos, productos, bienes y servicios, empleando para esta tarea las redes sociales, encuentros, intercambios, jornadas de formación permanente, desde las escuelas con los cierres de proyectos en la cual estudiantes, docentes y representantes muestras sus logros a los miembros de la comunidad escolar. Los foros universitarios donde los ponentes demuestran dominio, habilidades, destrezas, actitudes, aptitudes, logros, avances, experiencias producto de sus investigaciones.

\section{Incógnita, propósito y apología del estudio}

La individualidad hace que todos seamos diferentes, pero los puntos de coincidencia permiten el trabajo colectivo, al igual que el aprendizaje, se aprende en todo momento, todos los días, en cualquier situación, los docentes deben apropiarse de la didáctica como herramienta pedagógica, axiológica y metodológica, bajo esta premisa se genera la siguiente incógnita: ¿por qué la didáctica debe ser aprehendida en forma praxiológica para gestionar el conocimiento?

\section{Propósito del estudio}

Describir el pentagrama didáctico y su aproximación praxiológica de la gestión del conocimiento que facilita el proceso de aprendizaje individual y colectivo.

\section{Apología del estudio}

Se justifica esta producción intelectual, para generar una descripción de la didáctica, su aplicación praxiológica y las formas para gestionar el conocimiento a través de estrategias constructivas. Por su impacto educativo que facilita el aprendizaje desde una perspectiva innovadora. Para difundir reflexiones sobre la didáctica más allá de las aulas de clase, en espacios virtuales, con elementos musicales presentes y de fácil comprensión para los 
lectores. Por sus contenidos teórico prácticos que facilitan la enseñanza de cualquier disciplina artística, científica o de oficio.

\section{Moldura referencial del estudio}

En este marco de ideas, se consultaron los estudios internacionales que fundamentan esta investigación, Rodríguez (2014), desarrolló una tesis titulada: El aprendizaje autodidáctica en bibliotecas públicas y archivos históricos desde la perspectiva de Lev Vygotsky, en la Universidad de la Salle, Colombia, su objetivo general: "relacionar los planteamientos de Vigotsky, conceptos espontáneos, científicos y zonas de desarrollo próximo, con la función educativa de Bibliotecas Públicas y Archivos Históricos" (pág. 5); utilizó la metodología de investigación-acción, entre las conclusiones se destaca: "el trabajo educativo de la biblioteca pública mostró mayores facilidades para que una persona que no está asociada a la escolaridad y que pretenda realizar un proceso educativo de manera autónoma" (pág. 77).

Al promover la autonomía se relaciona con el presente estudio, las bibliotecas públicas ofrecen servicios educativos que permiten desarrollar en los usuarios un proceso cognitivo personalizado, fortaleciendo aspectos como la comprensión lectora, memoria, capacidad de análisis, sin estar directamente asociados a la educación formal, estos procesos personales, desarrollan el potencial creativo, destrezas y habilidades de investigación documental.

Asimismo, se cita a Curvelo (2016a), en su tesis titulada: Estrategias didácticas para el logro del aprendizaje significativo en los alumnos cursantes de la asignatura seguridad industrial, presentada en la Universidad de Carabobo, su objetivo general fue: "proponer un plan de estrategias didácticas para el logro del aprendizaje significativo en los alumnos cursantes de la asignatura seguridad industrial" (pág. 26). Enmarcada la investigación cuantitativa, bajo la metodología de proyecto factible, Curvelo (2016b), presentó como conclusiones: 
Los docentes no se han actualizado ni modificado, asumiendo como una de las razones para no incluir nuevas estrategias es la falta de recursos. El conocimiento de los docentes es escaso... tienen referencias pero no las han puesto en práctica (pág. 136).

Con relación al estudio, argumenta el autor, que el docente no cumple su rol de transformador a través del ejercicio de la profesión, su desactualización, falta de iniciativas creativas, excusas, sumado a currículos teóricos, debilitan el accionar del resto de los miembros del pentagrama didáctico (pág. 17). Es necesario retomar el papel protagónico del docente en todos los niveles y modalidades, para desarrollar clases dinámicas actualizadas, con estrategias didácticas motivadoras, sustanciosas de conocimientos teórico-práctico, aprovechando el máximo potencial del contexto de aprendizaje, talleres, áreas verdes, laboratorios, comedor, cantina, canchas deportivas, entre otras. Sin excusas, aplicando el plan B, C o $D$, en caso de contingencias, utilizar los recursos disponibles y es importante enseñar con el ejemplo.

\section{Constructivismo y acción comunicativa como podio teórico}

Seguidamente se presentan las teorías de fundamentan el presente estudio, Vygotsky (1985a), tiene entre las premisas que:

El individuo es el resultado de un proceso histórico y social, el constructivismo ayuda a los estudiantes a internalizar, reacomodar o transformar la información nueva, confeccionar significados, puntos de vista, ya que es un instrumento para el desarrollo cognitivo, apoyados con un adulto importante (pág. 31).

Somos el resultado del intercambio social e histórico, estamos en construcción permanente del conocimiento, afinamos procesos, hábitos de lectura e investigación, sensibilización, internalización, perspectivas, 
reajustes, construcción, deconstrucción y reconstrucción del contexto de aprendizaje. De igual forma, Vygotsky (1985b), destaca:

La zona de desarrollo próximo, en primer lugar como capacidad de resolución de situaciones o problemas, aquellas actividades realizadas independientemente por el estudiante. En segundo lugar, las que no puede realizar aún con ayuda. $Y$ finalmente, aquellas que caen en los dos extremos, las que puede realizar con ayuda de otros y las que no pueden ser realizadas a pesar de la ayuda (pág. 27).

Al realizar un ejercicio hermenéutico sobre la cita anterior, se puede agregar, las capacidades humanas superan en muchos aspectos situaciones y problemas, unas las hacen en forma independiente, otras con ayuda de otras personas, allí entra el papel de los docentes y familiares, el ritmo y estilo lo impone el mismo estudiante. Su autonomía le lleva a resolver la forma correcta de manipular un equipo tecnológico, sin necesidad de leer el manual o apoyo de un adulto, este caso es ampliamente conocido por la comunidad internacional. También, Vygotsky (1985c), considera:

El desarrollo humano es un proceso cultural, siendo la actividad del hombre el motor del proceso de desarrollo humano. El proceso de formación de las funciones pedagógicas superiores se dará a través de la actividad práctica e instrumental, pero no individual, sino en la interacción o cooperación social (pág. 36).

En consecuencia, el progreso humano es tangible desde distintos áreas culturales, cognitivas, deportivas, científicas, entre otras. Es el mismo individuo el motor fundamental de su propio desarrollo, al aprender, dominar funciones (básicas y complejas) pedagógicas, prácticas con diferentes herramientas e instrumentos, enmarcados en la interacción social, el intercambio de experiencias con otros individuos y el compartir datos e informaciones relevantes.

Agregando la teoría de la acción comunicativa propuesta por Habermas (1981a), destaca las premisas de regulación de normas, tales como lenguaje, 
lengua y habla, con manifestaciones simbólicas mediadoras en su interacción con el mundo, en la cual: "intervienen tres (3) procesos para la socialización en acción, con sus respectivos instrumentos y estrategias: 1.- Recepción y reproducción cultural. 2.- Integración social y resolución de problemas. 3.Desarrollo de la personalidad" (pág. 70). Los procesos comunicativos estudiados por Habermas, se centran en el accionar, con sus normas preestablecidas, incorpora los símbolos mediadores como gestos, señales para intercambiar mensajes con las personas del entorno y más allá de las fronteras en una acción socializadora en proceso.

Como proceso complejo intervienen instrumentos, estrategias, recursos, imágenes, señales que agrupó en tres grupos: La recepción y reproducción cultural permite apropiarnos de los mensajes cargados de conocimientos ancestrales e incorporarlos a los actuales. La integración social permite la resolución de conflictos, mediar en procesos para solventar diferencias y llegar a los acuerdos. El desarrollo de la personalidad es individual cada personal posee características propias que lo diferencias de los demás, según sus habilidades, destrezas, fortalezas comunicativas para el accionar en el entorno familiar, estudiantil, laboral, profesional y comunitario.

En tal sentido, Habermas (1981b), distingue dos modalidades de la acción comunicativa: "la acción instrumental y la acción estratégica (elección racional). La acción instrumental (técnicas y estrategias), se mantiene subordinada a las tradiciones legitimantes" (pág. 40). La acción comunicativa instrumental es la más utilizada sobre todo a nivel económico e intelectual, enmarcada en el cumplimiento de técnicas, normas, estrategias, pronunciación, reglas de redacción, oratoria y la misma cortesía. Por otra parte, la acción comunicativa estratégica involucra elegir racionalmente una postura frente al tema en discusión, perspectiva a favor o en contra, argumentar, confrontar, calificar, descalificar, aportar datos e informaciones actualizadas, es la más utilizada por los intelectuales y la sociedad del 
conocimiento.

\section{Alteraciones del pentagrama didáctico}

En esta parte se destacan los sostenidos, bemoles y becuadros que alteran el normal desarrollo de una nota en el pentagrama, son todos aquellos errores, omisiones, fallas, faltas, que causan la inconsistencia de la praxis didáctica, entre los elementos debilitantes y amenazantes se encuentran:

a). Falta de motivación: si algunos de los protagonistas del quehacer educativo están poco motivados o desmotivados, su actitud frente al conocimiento estará en riesgo de caer en la negatividad en el cumplimiento de objetivos, metas y competencias.

b). Inexactitud en la planificación: no es posible triunfar sin un plan, sin actividades claras, ni estrategias para lograrlo, el accionar exige una planificación por competencias, objetivos, propósitos, incluso de vida, los proyectos pueden ser a corto, mediano o largo plazo, con acciones contundentes, saber ¿qué hacer, como hacerlo y para que se hace un estudio?.

c). Errores en las prácticas: la falta de disciplina y hábitos de estudio diario generan un retardo en el perfeccionamiento de procesos básicos repetitivos, fórmulas, ejercicios, rutinas, postura, son chequeados en cada momento para reforzarlo, el mantener un bajo ritmo de trabajo afecta el rendimiento académico, escolar, laboral y otros, se recomienda hacerlos progresivamente lento, paso a paso, luego agregar velocidad y complejidad según la práctica a desarrollar.

d). Omitir pasos, datos e informaciones: los atajos no son buenos, aprender es siempre el objetivo, pero saltar procesos pueden repercutir negativamente en la forma, estilo, estructura y hasta las normas, por ejemplo: las reglas deportivas de cada disciplina, música de acuerdo a la vestimenta y el período histórico. 
e). Comentarios negativos e insultantes: denigrar de los estudiantes, hacerles actos de acoso escolar, malas jugadas, trampas en el accionar (conchitas de mango), hacer chistes sobre un error del compañero, podrían provocar hasta el retiro o un conflicto mayor. Son muchos los comentarios que los mismos docentes hacen de forma negativa de sus estudiantes, la mayoría en privado, pero, algunos lo hacen en público sin medir las consecuencias.

f). La falta de recursos, económicos, financieros, tecnológicos, humanos y materiales: que son cruciales para desempeñar las actividades y tareas, hacen que muchas metas no se cumplan. Debemos hacer uso correcto de los recursos disponibles, darle trato justo a los equipos e instrumentos de trabajo para obtener mayor vida útil de ellos.

\section{Praxiología de la gestión del conocimiento}

La Praxiología, es la ciencia que combina la teoría con la práctica y según Valhondo (2010), la gestión del conocimiento: "como se verá en su momento, comprender sus tradiciones es clave para interpretar sus actitudes y su manera de ser" (pág. 16). Como proceso permanente gestiona el conocimiento a través de unidades lógicas intelectuales, actividades inherentes a las tareas académicas identificar cantidades, objetos, instrumentos y personajes, encontrar la forma correcta de resolver problemas lógico matemáticos, clasificar datos e informaciones según un orden preestablecido (alfabético, numérico, talla, peso, cantidad, entre otros), presentar la ideas o productos intelectuales al resto de la comunidad.

Asimismo, Aquino, Barrios, Olivero y Mendoza (2017), define gestión del conocimiento como:

Una herramienta que permite implementar la estrategia competitiva de una organización, gobernando un proceso que consiste en: identificar, adquirir, almacenar, difundir, compartir, utilizar y actualizar el conocimiento tácito y explicito 
convirtiéndose así en una herramienta de aprendizaje y en un catalizador para la innovación (pág. 52).

En relación a la cita anterior, se destaca el vocablo herramienta de aprendizaje desbordada de estrategias competitivas como proceso cognitivo de utilidad académica, intelectual, organizativa, catalizador natural para la innovación, facilita adicionalmente técnicas, instrumentos, recursos, planificación y evaluación de lo aprehendido. Ejercitado a través de la práctica conducente al mejoramiento se transforma en un portal para el diseño, creación e innovación, estimula el pensamiento, las ideas, mejora la fluidez verbal, potencia la redacción, entre otros procesos intelectuales.

Los centros educativos desde la educación inicial hasta la universitaria deben procurar mejorar sus servicios educativos, instalaciones, actualizar currículos y tener un personal de planta que responda a la misión y visión organizacional. Los usuarios las diferentes casas de estudio deben apoyar las iniciativas para gestionar el conocimiento, con su participación activa, conformar equipos interdisciplinarios para hacer del quehacer educativo una experiencia enriquecedora para todos los miembros de la familia y comunidad en general. Los edificios y sus diferentes laboratorios puestos al servicio de la investigación práctica experimental donde los estudiantes puedan conocer a través de simuladores tecnológicos o experimentos complejos los aprendido teóricamente.

\section{Proyecciones visionarias del pentagrama didáctico}

Al medir el impacto del pentagrama didáctico desde dos tipos de investigación, cuantitativa y cualitativa, como estudio "expo facto", es reforzar o corroborar el contenido del presente estudio. Son muchas las investigaciones que demuestran la importancia de cada elemento presentado, en formas positivas y negativas. Se pretende con esta parte ir más allá de 
datos e informaciones, reales o forzadas por los investigadores, destacar el pentagrama didáctico como insumo para el crecimiento personal de la sociedad del conocimiento, para gestionar el conocimiento en forma consciente de los beneficios a corto, mediano y largo plazo. Afinar procesos tales como:

- Percepción del contenido programático de cada asignatura.

- Proyectar el conocimiento más allá del momento histórico.

- Visualizar la utilidad de lo aprehendido.

- Tener bases sólidas para comprobar o refutar teorías.

- Sentido de pertenencia y pertinencia.

- Mejoramiento continuo del proceso didáctico.

- Perfeccionar procesos científicos, artísticos o cualquier oficio.

- Producción innovadora, transformadora, científica, artística e intelectual.

\section{Reflexiones concluyentes}

Posterior al proceso de investigación y redacción del artículo, se presenta a continuación las reflexiones concluyentes, como punto que cierra un ciclo entre ustedes como lectores y el autor, en un diálogo intelectual virtual que tiene por finalidad las ideas o premisas finales que permitirán el juicio valorativo sobre la temática presentada. El pentagrama didáctico no es una figura geométrica cerrada con diferentes puntas y grados, son cinco líneas, cuatro espacios, claves, alteraciones e incluso aspectos generales ontológicos, metodológicos, axiológicos y praxiológicos que llevan a uno o más individuos a alcanzar el nivel de conocimiento esperado o mejor aún sobresalir en el dominio de un tema específico.

Preparen sus mentes, piensen en grande, no limiten sus actividades intelectuales, científicas, artísticas o de cualquier oficio, porque del 
conocimiento teórico y práctico, su dominio, utilización y aptitud, dependerá la evaluación o calificación obtenida. La mano de obra calificada es sólo una frase que describe el grado de maestría, habilidad, destreza, pericia, dominio que tiene un trabajador frente a la tarea a desarrollar.

Presta mucha atención a tus ideas, te abren otros caminos, nuevos procesos, innovación, crear, diseñar, inventar, descubrir y hasta improvisar para resolver problemas o situaciones laborales, familiares, académicas, deportivas, artísticas y de oficios. Busca la originalidad, agrega un estilo propio, ponles toques personales a tus tareas. No te conformes con un número de páginas mínimo, si lees más tendrá mayores oportunidades de producir como musas inspiradoras ideas cada vez más fascinantes.

Tus palabras son tu tarjeta de presentación, utiliza un léxico acorde a tu profesión u oficio, palabras técnicas especializadas, incluye en tus diálogos personajes, obras y fechas relevantes, por ejemplo, las efemérides del día como tema de conversación invita a los demás a prestarte mayor atención. Construye tus propios conceptos sobre la base de estudios previos y profundos, prepara tus discursos, ponencias, conferencias, practícalas una y otra vez, notarás que cada vez lo haces mejor. Escribe, redacta, publica notas, ensayos, artículos científicos para dar a conocer tus avances y logros a la sociedad del conocimiento.

El pentagrama didáctico es sólo una muestra del potencial humano y la gestión del conocimiento, permiten potenciar las capacidades del individuo culto, lectores, investigadores, estudiantes, docentes, a través de la praxiología combinar en diferentes porcentajes (\%) la teoría con la práctica, sin limitaciones por la edad, sexo, condición social y económica. Todos los elementos se acoplan para vencer la sombra de la ignorancia, desconocimiento, torpeza e inexperiencia, para transformar a un aprendiz en un maestro con habilidades, conocimientos y prácticas que satisfacen las expectativas de sus clientes. 
Como alternativa para mejorar tu nivel de aprendizaje, aprópiate del contenido del pentagrama didáctico, lee y relee, práctica una y otra vez, toma el control de tu propio proceso cognitivo, aumenta las horas de estudio en forma progresiva, investiga más allá de las pautas preestablecidas por los docentes, personaliza tus láminas, agrega datos e informaciones relevantes a tus conversaciones, ponencias o conferencias. Tú puedes hacer la diferencia como docente o estudiante, aporta, agrega, da más de lo esperado.

Exige más calidad educativa, pero haciendo tu parte, el sistema educativo es papel escrito, quienes lo ejecutan son los encargados de darle valor a las letras, contenidos y actividades que busquen el mejoramiento permanente del individuo, en la cual el docente es sólo una pieza del rompecabezas, estudiantes, familiares, comunidad y el currículo son algunos de los elementos que hemos tocado usted puede agregar muchos más, hacerlo más complejo pero, al final del proceso la producción intelectual es la diferencia entre el bueno y el mejor.

La práctica es el mejor de los procesos para gestionar el aprendizaje, las experiencias significativas, constructivas, dirigidas y vivenciales hacen que todo estudiante florezca sus capacidades innatas, vocación de servicio, habilidades, destrezas, es decir, todo lo bueno, pero, siempre y cuando el estudiante aprenda, con el docente, sin el docente y a pesar del docente.

\section{Referencias}

Aquino, B., Barrios, K., Olivero, E., \& Mendoza, J. (2017). Gestión del conocimiento y capacidades de innovación en una Organización Radial en Barranquilla. Barranquilla, Colombia: Ediciones Universidad Simón Bolívar. Recuperado de:

https://bonga.unisimon.edu.co/bitstream/handle/20.500.12442/2089/Ca

p 7 Gesti\%C3\%B3nConocimiento. pdf?sequence=11\&isAllowed=y

Curvelo, D. (2016a,b). Estrategias didácticas para el logro del aprendizaje 
significativo en los alumnos cursantes de la asignatura seguridad industrial. Tesis de especialización. Bárbula, Carabobo, Venezuela: Universidad de Carabobo. Recuperado de:

http://riuc.bc.uc.edu.ve/bitstream/123456789/3878/1/dcurvelo.pdf

Díaz, (2001). Metodología cualitativa. Madrid, España: Editorial Océano.

Diccionario de las Ciencias de la Educación (1983). Didáctica. Volumen 1, ISBN 10: 8429420711; ISBN 13: 9788429420715. Editorial España: Diagonal/Santillana, S.A.

Habermas, J. (1981a,b), Teoría de la acción comunicativa. España: Editorial McGregor.

Madrid, E., \& Mayorca, A. (2010). Didáctica como disciplina pedagógica. Madrid, España: Ediciones Cincel.

Moreno, I. (2004). La Utilización de Medios y Recursos Didácticos en el Aula. Madrid, España: Facultad de Educación de la Universidad Complutense de Madrid. Recuperado de:

https://webs.ucm.es/info/doe/profe/isidro/merecur.pdf

Rodríguez, C. (2014). El Aprendizaje Autodidacta en Bibliotecas Públicas y Archivos Históricos desde la Perspectiva de Lev Vygotsky. Tesis. Bogotá, Colombia: Universidad de la Salle. Recuperado de: http://repository.lasalle.edu.co/bitstream/handle/10185/20702/7412120 2 2014.pdf? sequence $=1$ \&isAllowed $=y$

Valhondo, D. (2010). Gestión del Conocimiento: Del Mito a la Realidad. ISBN: 978-84-7978-195-8. Madrid, España: Ediciones Díaz de Santos, S.A.

Vygotsky, L. (1985a,b,c). Pensamiento y lenguaje. Buenos Aires. Argentina: Editorial Pléyade.

Wolfgang, M. (1929). Principios de la didáctica. Madrid, España: Editorial Anaya. 


\section{Pedro Jacobo Caldera Tovar}

e-mail: drjacobocaldera@gmail.com

Venezolano, nací en San Felipe, estado Yaracuy, el 2 de

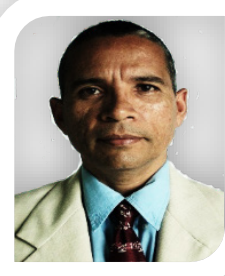
abril del año 1970. Soy Profesor en educación técnica, del Instituto Universitario Pedagógico Monseñor Arias (IUPMA); Especialista en gerencia educativa, de la Universidad Experimental Libertador (UPEL); Doctor en Ciencias de la Educación, de la Universidad Fermín Toro (UFT); Doctor en innovaciones educativas, de la Universidad Nacional Experimental de las Fuerzas Armadas (UNEFA); Postdoctor en estudios libres, de la Universidad Fermín Toro (UFT); Además de músico, cantante, compositor, saxofonista, clarinetista y director de los Antaños del Yara.

El contenido de este manuscrito se difunde bajo una Licencia de Creative Commons ReconocimientoNoComercial-Compartirlgual 4.0 Internacional 